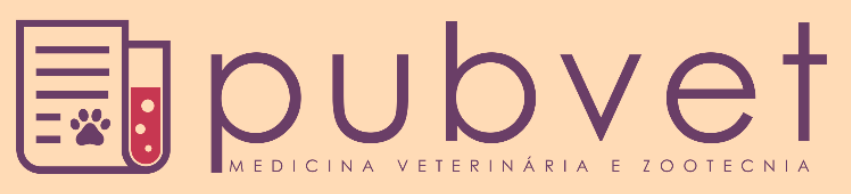

https://doi.org/10.31533/pubvet.v14n10a661.1-6

\title{
Avaliação analgésica da metadona, em duas doses associada a lidocaína sem vasoconstritor, por via epidural em cães submetidos a orquiectomia eletiva
}

\author{
Henrique Jorge Vieira Antunes Junior ${ }^{1 *} \bullet$, Natália Yoshioka de Vidis ${ }^{2} \bullet$, Alessandra Augusto Bairros ${ }^{3} \bullet$ \\ ${ }^{I}$ Aluno do programa de aprimoramento em Medicina Veterinária - UNIDERP/Anhanguera \\ ${ }^{2}$ Professora do curso de Graduação de Medicina Veterinária - UNIDERP/Anhanguera \\ ${ }^{3}$ Medica Veterinária da Clínica Veterinária UNIDERP/Anhanguera \\ *Autor para correspondência, E-mail: hantunesjr@hotmail.com
}

Resumo. Diversos fármacos podem ser empregados no controle da dor, por diversas vias, como a epidural. A metadona é um opioide sintético semelhante a morfina, podendo ser utilizado pela via epidural para controle da dor, principalmente durante procedimentos cirúrgicos como a orquiectomia. Foram selecionados nove cães machos para realização do estudo de ação analgésica da metadona por via epidural em duas concentrações, $0,1 \mathrm{mg} / \mathrm{kg}$ (M01) e 0,3 mg/kg (M03), associadas a lidocaína sem vasoconstritor, o grupo controle recebeu apensas lidocaína (LID) por via epidural. Para avalia os grupos foram mesurados e analisados estatisticamente os valores de frequência cardíaca, frequência respiratória, saturação de oxigênio, temperatura e pressão arterial sistólica. Os grupos M01 e M03 não divergiram entre si estatisticamente, o grupo LID apresentou medias diferentes dos grupos M01 e M03 para os valores de frequência cardíaca e respiratória, saturação de oxigênio e pressão arterial diastólica, confirmando a necessidade da utilização de fármacos analgésicos em procedimentos cirúrgicos, principalmente nos momentos de maior estimulação nociceptiva.

Palavras-chave: Anestesiologia, analgesia, castração

\section{Analgesic evaluation of methadone, in two doses associated with lidocaine without vasoconstrictor, epidurally in dogs submitted to elective orchiectomy}

\begin{abstract}
Several drugs have used to control pain through various routes, such as epidural. Methadone is a synthetic morphine-like opioid and has used epidurally for pain control, especially during surgical procedures such as orchiectomy. Nine male dogs were selected for epidural methadone analgesic action study in two concentrations, $0.1 \mathrm{mg} / \mathrm{kg}(\mathrm{M} 01)$ and $0.3 \mathrm{mg} / \mathrm{kg}$ (M03), associated with lidocaine without vasoconstrictor, the control group was appended. epidural lidocaine (LID). To evaluate the groups were measured and statistically analyzed the values of heart rate, respiratory rate, oxygen saturation, temperature and systolic blood pressure. The groups M01 and M03 did not differ statistically, the LID group presented different means of the groups M01 and M03 for the values of heart rate, oxygen saturation and diastolic blood pressure, confirming the need to use analgesic drugs in surgical procedures, especially at times of increased nociceptive stimulation.
\end{abstract}

Keywords: Anesthesiology, analgesia, castration 


\title{
Evaluación analgésica de la metadona, en dos dosis asociadas a la lidocaína sin vasoconstrictor, por vía epidural en perros sometidos a castración electiva
}

\begin{abstract}
Resumen. Diversos fármacos pueden ser empleados en el control del dolor por diferentes vías como la epidural. La metadona es un opioide sintético, similar a la morfina y puede ser utilizado por la vía epidural para el control del dolor, principalmente durante procedimientos quirúrgicos como la castración. Fueron seleccionados nueve perros machos para la realización del estudio de la acción analgésica de la metadona por vía epidural en dos concentraciones, $0.1 \mathrm{mg} / \mathrm{kg}$ (M01) y $0.3 \mathrm{mg} / \mathrm{kg}$ (M03), asociada a la lidocaína sin vasoconstrictor, el grupo control recibió lidocaína (LID) por vía epidural solamente. Para evaluar los grupos fueron medidos y analizados estadísticamente los valores de frecuencia cardiaca, frecuencia respiratoria, saturación de oxígeno, temperatura y presión arterial sistólica. Los grupos M01 y M03 no tuvieron diferencias significativas en las variables estudiadas, el grupo LID presentó promedios diferentes de los grupos M01 e M03 para los valores de frecuencia cardíaca y respiratoria, saturación de oxígeno e presión arterial diastólica, confirmando la necesidad de la utilización de fármacos analgésicos en procedimientos quirúrgicos, principalmente en los momentos de mayor estimulación nociceptiva.
\end{abstract}

Palabras clave: Anestesiología, analgesia, castración

\section{Introdução}

Opioides são agentes terapêuticos amplamente empregados na medicina veterinária para controle da dor pós-operatória, pois possuem efeitos analgésicos e sedativos, podendo ser administrados por diferentes vias e promovendo assim diversos graus de analgesia, podendo mudar de acordo com o agente e dose utilizado (Pascoe, 2000; Valadão et al., 2002). Diversos estudos têm ressaltado o uso da epidural com opioides, sendo uma excelente alternativa no controle da nocicepção em animais, no pósoperatório principalmente, produzindo analgesia pós-cirúrgica dose dependente e de melhor qualidade e maior duração quando comparada a administração destes agentes por outras vias, como a parenteral (Leibetseder et al., 2006; Pekcan \& Koc, 2010; Polydoro et al., 2006).

De acordo com Valadão et al. (2002), a associação de anestésicos locais e opioides por via epidural, promovem analgesia com a redução dos efeitos colaterais quando comparado ao uso isolado destes fármacos. Além disso, alguns estudos apontam que esta associação promovem a extensão da analgesia a segmentos espinhais mais craniais, com maior duração do bloqueio sensitivo, sem aumentar o bloqueio motor promovido pelo anestésico local (Cassu et al., 2010; Polydoro et al., 2006; Wetmore \& Glowski, 2000).

A metadona é um opioide sintético, que apresenta efeitos farmacológicos semelhantes à morfina, sendo um agonista dos receptores opioides mi $(\mu)$, delta $(\delta)$ e kappa $(\kappa)$ que, paralelamente, atua como antagonista dos receptores $\mathrm{N}$-metil D-aspartato (NMDA), o que contribui para incrementar a analgesia promovida por este fármaco, principalmente no tratamento de dor crônicas e neuropática(Bley et al., 2004; Snyder et al., 1980). Apresenta vantajosas características que incluem período de ação prolongado e potência analgésica alta, sem a formação de metabólitos ativos (Lamont \& Mathews, 2007).

\section{Material e métodos}

Foram utilizados nove cães machos, sem raça definida, com idade média de três anos e peso variado, que seriam submetidos a orquiectomia eletiva. A seleção dos animais foi baseada no exame clínico, hemograma completo e exames bioquímicos com valores fisiológicos dentro dos limites considerados normais para a espécie. Foram descartados do estudo animais que apresentaram alterações nos exames, deformidade na pelve ou coluna vertebral e obesos. Os animais passaram por jejum alimentar de 12 horas, sem restrição hídrica. 
Foi estabelecido três grupos para execução do experimento, o primeiro grupo que receberia anestesia epidural com metadona na dose $0,1 \mathrm{mg} / \mathrm{kg}$ (M01) associada a lidocaína sem vasoconstritor, o segundo grupo, que receberia metadona na dose de $0,3 \mathrm{mg} / \mathrm{kg}$ (M03) associada a lidocaína sem vasoconstritor, que correspondem aos dois grupos estudos e um grupo controle, o terceiro grupo, que recebeu epidural com lidocaína sem vasoconstritor (LID), sendo que o volume final ficou estabelecido em $1 \mathrm{ml}$ a cada $4,5 \mathrm{~kg}$, para os três grupos.

Para padronização do estudo, foi realizado um cronograma dos momentos em que seriam realizadas as aferições dos parâmetros (Figura 1), que foi realizado por um único avaliador para minimizar possíveis erros de aferições.

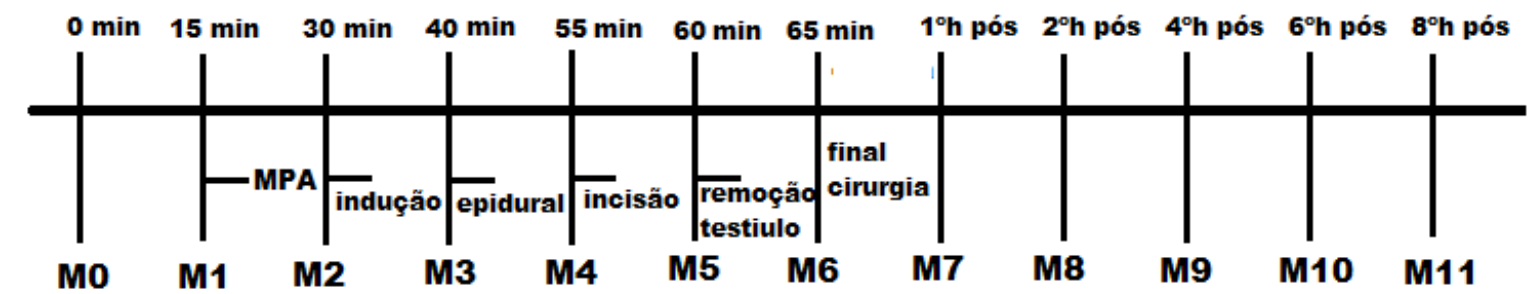

Figura 1. Linha do tempo, onde foram estabelecidos os momentos (M) de aferições dos parâmetros avaliados, onde M0 corresponde o início do experimento e primeira aferição dos parâmetros e M11 o último momento de aferição, que corresponde a 8 horas após o término da cirurgia.

No dia do experimento os animais foram divididos aleatoriamente entre os grupos, foram aferidos os parâmetros basais (M0) e posteriormente foram realizadas as tricotomias e o cateterismo venoso e fluido terapia com ringer lactato, ambos os grupos receberam como medicação pré-anestésica (MPA) acepromazina na dose de $0,06 \mathrm{mg} / \mathrm{kg}$ por via intramuscular e cefalotina sódica na dose de $25 \mathrm{mg} / \mathrm{kg}$ por via endovenosa, após quinze minutos foi realizada a segunda aferição dos parâmetros (M1), os animais foram induzidos com propofol na dose de $4 \mathrm{mg} / \mathrm{kg}$ por via endovenosa e o mantidos em plano anestésico com anestesia inalatória com isoflurano com sonda endotraqueal, após a tingir o plano anestésico foi realizada a terceira aferição dos parâmetros (M2), posteriormente foram mantidos em decúbito esternal e realizada a antissepsia para realização da epidural no espaço intervertebral da L7 e S1 estabilizandoos nessa posição por quinze minutos e posteriormente foi realizada a quarta aferição (M3), subsequente a este momento os animais foram colocados em decúbito lateral direito dando início ao procedimento cirúrgico. Após a realização da antissepsia da bolsa escrotal foi realizada a aferições nos seguintes momentos, incisão da bolsa escrotal (M4), remoção do segundo testículo (M5) e término da dermorrafia (M6).

Os pacientes foram avaliados novamente uma hora (M7), duas horas (M8), quatro horas (M9), seis horas (M10) e oito horas após a cirurgia (M11), subsequente a esse momento foi administrado meloxicam na dose de $0,2 \mathrm{mg} / \mathrm{kg}$ e cloridrato de tramadol na dose $\mathrm{de} 4 \mathrm{mg} / \mathrm{kg}$ ambos por via endovenosa.

Os parâmetros avaliados foram frequência cardíaca (FC), frequência respiratória (FR), temperatura retal (T), pressão arterial sistólica (PS) aferida com esfignomanômetro digital e valores de oximetria que foram aferidos com oxímetro lingual $\left(\mathrm{PO}_{2}\right)$.

Para realização das análises estatísticas foi utilizado o programa GraphPad Prism 8, realizando análise de variância (ANOVA) para amostras não-pareadas, com posterior teste de Tukey, para comparações de medias dentro de cada grupo em relação aos valores basais. As diferenças foram consideradas significantes somente quando $\mathrm{P}<0,05$. Todos os resultados foram expressos em média \pm desvio padrão.

\section{Resultados e discussão}

Todos os animais obtiveram grau de sedação excelente durante todo o transcirurgico. O grupo M01 apresentou menor alteração na FC e o grupo LID apresentou maior desvio padrão quando comparado aos demais grupos (Figura 2). 
Os efeitos dos opioides sobre o sistema cardiovascular dependem do fármaco utilizado, da velocidade de aplicação e da dose empregada, toda via a diminuição da frequência cardíaca é o efeito mais comum quando opioides $\mu$ agonista são utilizados (Otero \& Portela, 2018). Quando comparado com os grupos M01 e M03, o grupo LID apresentou maior diferença nas alterações da FC (Figura 3).

\section{Frequencia cardiaca (FC) media de cada grupo com o desvio padrão.}

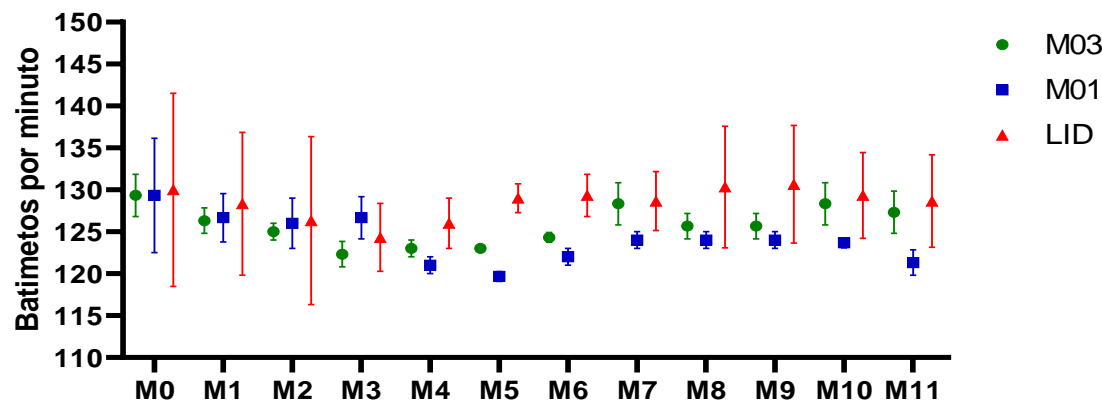

Figura 2. Representação gráfica da média e desvio padrão. O grupo LID apresentou maior desvio, nota-se o aumento da FC nos momentos M4, M5 e M6, onde se tem maior estímulo neurogênico.

Os dados gasométricos obtidos nos momentos de M2 a M6 apontam que o consumo de oxigênio e dor estão correlacionados (Tabela 1), juntamente com o aumento da FC, enquanto a $\mathrm{PO}_{2}$ diminui a FC aumenta. De acordo com Muir \& Gaynor (2009), uma das consequências fisiopatológicas da dor é a taquicardia e o consumo de oxigênio aumentado, assim como a hipertensão, vasoconstrição e trabalho cardíaco.

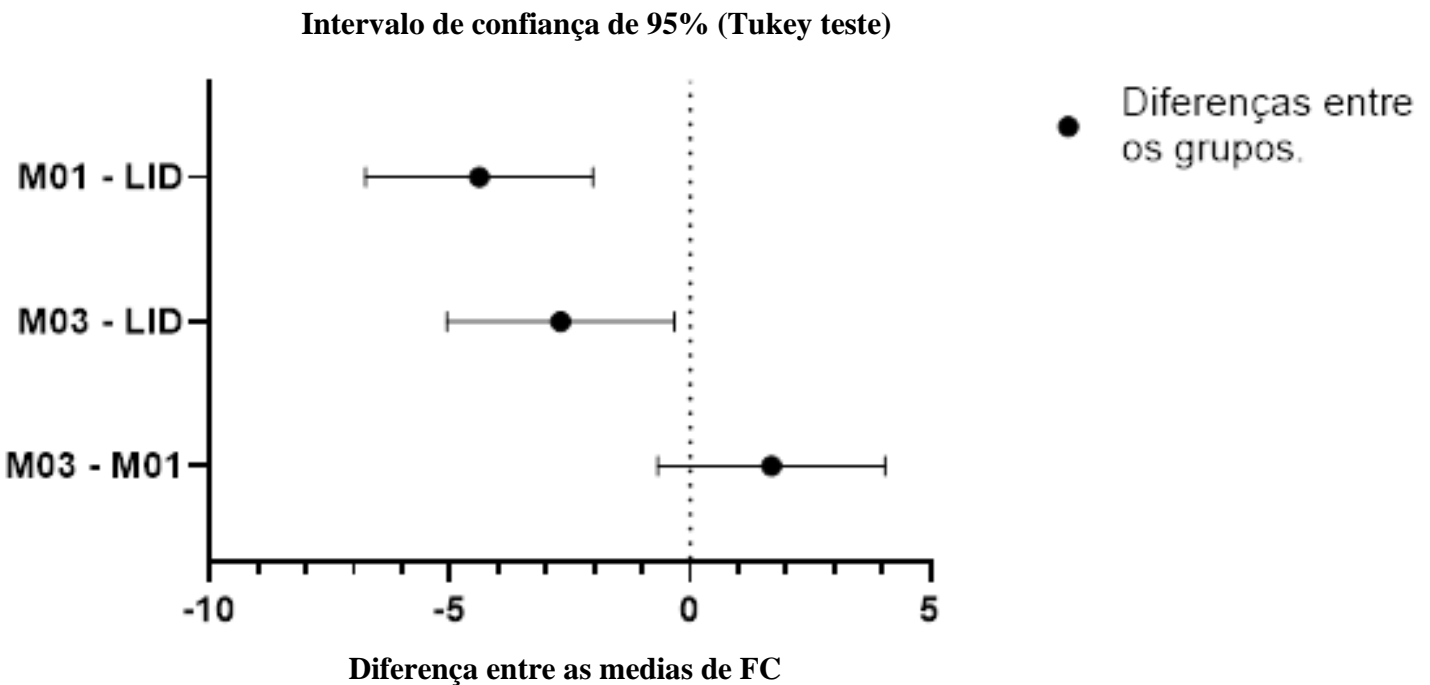

Figura 3. Representação gráfica do teste de Tukey para os valores de FC dos grupos estudados. Observa-se que o grupo LID quando comparado com os outros grupos apresenta resultado dentro do intervalo de confiança.

Tabela 1. Comparação dos valores de gasometria entre os grupos, levando em consideração os valores de média e desvio padrão de cada grupo.

\begin{tabular}{lccc}
\hline Teste de Tukey & Diferença das medias & Intervalo de Confiança 95\% & Significativo? \\
\hline M01 vs. M03 & 0,5333 & $-0,4134$ a 1,480 & Não \\
M01 vs. LID & 2,067 & 1,120 a 3,013 & Sim \\
M03 vs. LID & 1,533 & 0,5866 a 2,480 & Sim \\
\hline
\end{tabular}

No transoperatório, ocorreu a diminuição da frequência respiratória em relação ao basal, essa diferença foi observada em todos os grupos, principalmente nos momentos de M2 a M7, independentemente do tratamento submetido, entretanto através das análises não foi computada 
significância estatísticas entre quaisquer grupos (Tabela 2). De acordo com Garofalo, (2010) e Campagnol (2011), essa é uma alteração comum, decorrente do isoflurano, um dos fármacos empregados na anestesia, que causa decréscimo da frequência respiratória. Assim como a acepromazina que é conhecida por causar comumente esta alteração (Mastrocinque \& Fantoni, 2003; Mastrocinque, 2005). Os opioides também promovem depressão acentuada da frequência respiratória, citado até como um dos principais efeitos colaterais (Keegan et al., 1995).

Tabela 2. Frequência respiratória (FR), representada em média \pm desvio de cada grupo a cada momento

\begin{tabular}{lllllllllllll}
\hline & M0 & M1 & M2 & M3 & M4 & M5 & M6 & M7 & M8 & M9 & M10 & M11 \\
\hline M01 & $46.7 \pm 1.5$ & $43.7 \pm 2.5$ & $27.3 \pm 2.5$ & $25.0 \pm 2.6$ & $25.0 \pm 1.0$ & $24.7 \pm 0.6$ & $27.3 \pm 0.6$ & $41.3 \pm 3.5$ & $42.0 \pm 1.0$ & $32.7 \pm 2.0$ & $30.7 \pm 0.6$ & $33.3 \pm 0.6$ \\
M03 & $43.3 \pm 2.1$ & $42.7 \pm 3.2$ & $31.3 \pm 1.5$ & $26.0 \pm 4.6$ & $25.3 \pm 0.6$ & $24.0 \pm 1.0$ & $24.7 \pm 1.2$ & $34.3 \pm 3.8$ & $36.7 \pm 3.2$ & $36.7 \pm 2.1$ & $36.3 \pm 3.5$ & $34.7 \pm 2.3$ \\
LID & $48.7 \pm 3.1$ & $36.3 \pm 2.1$ & $30.7 \pm 3.1$ & $24.7 \pm 2.5$ & $29.0 \pm 3.6$ & $29.3 \pm 2.1$ & $29.7 \pm 0.6$ & $42.7 \pm 3.5$ & $41.7 \pm 2.1$ & $35.7 \pm 4.2$ & $33.0 \pm 3.0$ & $34.0 \pm 2.6$ \\
\hline
\end{tabular}

* Não foi observado relação de significância estatística nas variações observadas entre grupos.

Assim como os valores de $\mathrm{FC}$ e $\mathrm{PO}_{2}$, os valores da pressão arterial sistólica (PAS) foram aferidos e apresentaram significância estáticas, quando comparados os grupos M01 ou M03 com o grupo LID, novamente nos momentos de M4 a M6 ocorreram o maior aumento nos valores médios da PAS (Tabela 3).

Tabela 3. Resultado do teste de Tukey para os valores de PAS, dos grupos M01, M03 e LID.

\begin{tabular}{lccc}
\hline Teste de Tukey & Diferença das medias & Intervalo de confiança 95\% & Significativo? \\
\hline M01 vs. M03 & $-0,3056$ & $-2,812$ a 2,201 & Não \\
M01 vs. LID & $-4,444$ & $-6,951$ a $-1,938$ & Sim \\
M03 vs. LID & $-4,139$ & $-6,646$ a $-1,632$ & Sim \\
\hline
\end{tabular}

As alterações de aumento na pressão arterial sistólica está intimamente ligada aos momentos de maior estimulo doloroso (Muir \& Gaynor, 2009). De acordo com Campagnol (2011) a metadona quando administrada por via epidural pode promover uma significativa redução nos valores de PAS, o que não foi observado nesse experimento.

\section{Conclusão}

A metadona promove analgesia significativa, com o mínimo de efeitos colaterais, o que a torna um excelente fármaco para o controle da dor, principalmente decorrente a estímulos dolorosos de procedimentos cirúrgicos como por exemplo a orquiectomia, principalmente durante o procedimento onde ocorre maior estímulo nociceptivo. Não existiram variações estatísticas entre as doses usadas no experimento, isso permite a utilização de uma dose menor, com menor risco e melhor custo-benefício, sem redução da analgesia durante a orquiectomia.

\section{Referências bibliográficas}

Bley, C. R., Neiger-Aeschbacher, G., Busato, A., \& Schatzmann, U. (2004). Comparison of perioperative racemic methadone, levo-methadone and dextromoramide in cats using indicators of post-operative pain. Veterinary Anaesthesia and Analgesia, 31(3), 175-182. DOI: https://doi.org/10.1111/j.1467-2987.2004.00148.x

Campagnol, D. (2011). Farmacologia clínica da metadona peridural e intravenosa em cães. Universidade Estadual Paulista (UNESP).

Cassu, R. N., Melchert, A., Silva, A. P. G. da, Reis, A. M. dos, \& Meirelles, C. C. (2010). Lidocaína com vasoconstrictor isolada e associada ao fentanil via peridural em cães. Ciência Rural, 40(3), 580586. DOI: https://doi.org/10.1590/s0103-84782010005000016

Garofalo, N. A. (2010). Alterações hemodinâmicas e neuroendócrinas associadas ao uso da metadona em cães conscientes e anestesiados com isoflurano. Universidade Estadual Paulista (UNESP).

Keegan, R. D., Greene, S. A., \& Weil, A. B. (1995). Cardiovascular effects of epidurally administered morphine and a xylazine-morphine combination in isoflurane-anesthetized dogs. American Journal of Veterinary Research, 56(4), 496-500. 
Lamont, L. A., \& Mathews, K. A. (2007). Opioids, nonsteroidal anti-inflammatories, and analgesic adjuvants. Lumb \& Jones' Veterinary Anesthesia and Analgesia, 4, 241-271.

Leibetseder, E. N., Mosing, M., \& Jones, R. S. (2006). A comparison of extradural and intravenous methadone on intraoperative isoflurane and postoperative analgesia requirements in dogs. Veterinary Anaesthesia and Analgesia, 33(2), 128-136. DOI: https://doi.org/10.1111/j.14672995.2005.00239.x

Mastrocinque, S, \& Fantoni, D. T. (2003). A comparison of preoperative tramadol and morphine for the control of early postoperative pain in canine ovariohysterectomy. Veterinary Anaesthesia and Analgesia, 30(4), 220-228. DOI: https://doi.org/10.1046/j.1467-2995.2003.00090.x

Mastrocinque, S. (2005). Avaliação do emprego do tramadol epidural ou sistêmico e da morfina epidural em cadelas submetidas à ovariohisterectomia. Universidade de São Paulo.

Muir, W. W., \& Gaynor, J. S. (2009). Manual de controle da dor em medicina veterinária (Vol. 1). MedVet.

Otero, O. E., \& Portela, D. A. (2018). Anestesia regional em animais de estimação. Editora MedVet.

Pascoe, P. J. (2000). Opioid analgesics. Veterinary Clinics: Small Animal Practice, 30(4), 757-772.

Pekcan, Z., \& Koc, B. (2010). The post-operative analgesic effects of epidurally administered morphine and transdermal fentanyl patch after ovariohysterectomy in dogs. Veterinary Anaesthesia and Analgesia, 37(6), 557-565. DOI: https://doi.org/10.1111/j.1467-2995.2010.00571.x

Polydoro, A. S., Natalini, C. C., Raiser, A. G., \& Hennemanin, C. R. A. (2006). Analgesia e anestesia epidural em cães e gatos: revisão de literatura. A Hora Veterinária, 25(149), 31-37.

Snyder, E. W., Shearer, D. E., Schlehuber, C., Dustman, R. E., \& Beck, E. C. (1980). Prolonged electrophysiological and behavioral alterations following a single injection of methadone in the cat. Pharmacology Biochemistry and Behavior, 12(6), 893-898. DOI: https://doi.org/10.1016/0091$\underline{3057(80) 90450-5}$

Valadão, C. A. A., Duque, J. C., \& Farias, A. (2002). Administração epidural de opióides em cães. Ciência Rural, 32(2), 347-355. DOI: https://doi.org/10.1590/s0103-84782002000200028

Wetmore, L. A., \& Glowski, M. M. (2000). Epidural analgesia in veterinary critical care. Clinical Techniques in Small Animal Practice, 15(3), 177-188.

Licenciamento: Este artigo é publicado na modalidade Acesso Aberto sob a licença Creative Commons Atribuição 4.0 (CC-BY 4.0), a qual permite uso irrestrito, distribuição, reprodução em qualquer meio, desde que o autor e a fonte sejam devidamente creditados. 